\title{
Antioxidant, iron chelating, lipase inhibition activities and metabolite's prediction of hyrdoethanolic leaf extract of Conocarpus erectus
}

\author{
1,* Raza, S.A., ${ }^{1}$ Chaudhary, A.R., ${ }^{2}$ Mumtaz, M.W. and ${ }^{3}$ Bashir, S. \\ ${ }^{1}$ Department of Chemistry, Government College University, Lahore, Pakistan \\ ${ }^{2}$ Department of Chemistry, University of Gujrat, Pakistan \\ ${ }^{3}$ Division of Science and Technology, University of Education, College Road Lahore, Pakistan
}

\begin{abstract}
Article history:
Received: 22 August 2019

Received in revised form: 16

September 2019

Accepted: 18 September 2019

Available Online: 14 October 2019
\end{abstract}

Keywords:

Antioxidant,

Fe-chelating,

Lipase inhibition,

Ultrasonication,

Conocarpus erectus,

${ }^{1} \mathrm{H}-\mathrm{NMR}$.

DOI:

https://doi.org/10.26656/fr.2017.4(2).300

\begin{abstract}
The current study was conducted to evaluate the antioxidant, Fe-chelating and lipase inhibition activities of hydroethanolic leaf extracts of Conocarpus erectus. The proton magnetic resonance spectroscopy $\left({ }^{1} \mathrm{H}-\mathrm{NMR}\right)$ was used to identify the major types of primary and secondary metabolites in extract. The $60 \%$ ethanolic extract was found to be the most effective fraction regarding antioxidant, Fe chelating and lipase inhibitory properties. The $60 \%$ ethanolic leaf extract exhibited total antioxidant power of $223.0 \pm 3.27$ $\mathrm{mg}$ ASE/g PE, $\beta$-carotene bleaching inhibition of $81.39 \pm 2.11 \%$, Fe-chelating of $68.21 \pm 1.17 \%$ and pancreatic lipase inhibition of $50.60 \pm 1.47 \%$, respectively. The ${ }^{1} \mathrm{H}-\mathrm{NMR}$ -based prediction of metabolites provided the information for presence of polyphenolic secondary metabolites and organic acids which might be responsible for the biological activities of extract. These findings of this work may be extended for metabolite characterization, in vivo trials and toxicity determination to get benefits for functional food development with antioxidative and antiobesity attributes.
\end{abstract}

\section{Introduction}

Reactive oxygen species (ROS) are produced in the human body as result of metabolic functions and play an important role in cell signaling. The overproduction of ROS is counterbalanced by antioxidant defense system of body. Any imbalance in ROS and antioxidants may create a situation of oxidative stress which involves initiation and progression of many chronic health disorders. The antioxidant intake is considered an effective tool to mitigate and manage oxidative stress and stress-related disorders like diabetes, cancer, obesity, aging and many others (Piyush et al., 2010; William et al., 2018). The commercially available synthetic antioxidants are although very effective but pose serious threats to human health due to their toxic nature. Commonly used synthetic antioxidants like butylated hyroxyanisole (BHA), butylated hydroxytoluene (BHT) and ter-butyl hydroquinone (TBHQ) have been proved toxic (Madhujith and Shahidi, 2006; Wang et al., 2010). There are still many concerns about the safety of synthetic antioxidants which are being monitored regularly in Europe and developed countries.

Under such circumstances, plants provide a wide range of opportunities for availability of potent antioxidants of safe and therapeutic nature. The plant extracts rich in polyphenols were reported to have notable antioxidant and enzyme inhibition properties. These activities were indicators of possible medicinal attributes of plants (Raza et al., 2013; William et al., 2018). The iron-chelating activity of plant extracts is reported as efficient strategy to mitigate the harmful impacts of overloaded iron in body. The iron overload is involved in production of ROS, initiation and progression of diabetes along with damage to liver function (Shaaban et al., 2016). Similarly, pancreatic lipase inhibition is a useful way to reduce the burden of obesity. Plants have been reported as a natural source for safe metal chelating and lipase inhibitory agents. The antioxidants present in plants were mentioned as potent metal chelators and natural inhibitors of pancreatic lipase.

Concarpus erectus (Combretaceae) has gained importance due to recently explored medicinal aspects including antidiabetic, antimicrobial, anticancer and hepatoprotective effects (Saleh et al., 2012; Saleh et al., 2013; Nascimento et al., 2016; Raza et al., 2018). The metal chelating activity and pancreatic lipase inhibitory properties of $C$. erectus have not been reported previously as per best of available information. 
Therefore, the current work was performed to evaluate the antioxidant activity, iron-chelating activity, pancreatic lipase inhibition potential of $C$. erectus leaf extracts along with ${ }^{1} \mathrm{H}-\mathrm{NMR}$ based metabolite prediction.

\section{Material and methods}

\subsection{Hydroethanolic extraction}

The $C$. erectus leaves were identified from the Department of Botany, GCU Lahore vide voucher specimen GC.Herb.33.Bot. 3379. Fresh leaves were collected and immediately quenched with liquid nitrogen, lyophilized and suspended in solvent systems (aqueous, 20\% ethanol, 40\% ethanol, 60\% ethanol and pure ethanol). The samples were ultrasonicated at Soniprep 150, filtered and extra solvent was removed on rotary evaporator under reduced pressure. The extracts were stored at low temperature until further use.

\subsection{Total antioxidant power}

Total antioxidant activity of all extract fractions was based on phosphomolybdenum complex formation methods with little modification as previously reported (Prieto et al., 1999). Briefly, $250 \mu \mathrm{g} / \mathrm{mL}$ of each extract was mixed with $4 \mathrm{~mL}$ of reagent solution consisting of $0.6 \mathrm{M}$ sulphuric acid, $4 \mathrm{mM}$ ammonium molybdate and $28 \mathrm{mM}$ sodium phosphate in capped plastic vials. The obtained mixtures along with a blank solution $(4 \mathrm{ml}$ reagent solution) were incubated in water bath at $95^{\circ} \mathrm{C}$ for 90 mins. After cooling to room temperature, absorbance was noted at $695 \mathrm{~nm}$. A standard curve was drawn using ascorbic acid. The butylated hyroxyanisole (BHA) was used as standard antioxidant for comparison of antioxidant potential of extracts. The results were represented as ascorbic acid equivalent per gram dried plant extract (ASE/g DE).

\section{$2.3 \beta$-carotene bleaching assay}

Antioxidant activity of $C$. erectus leaf extracts was determined by assessing the bleaching of $\beta$-carotene (yellow color) in the presence of linoleic acid (Shon et al., 2003). Initially, $2 \mathrm{mg}$ of $\beta$-carotene were dissolved in $10 \mathrm{~mL}$ of chloroform followed by subsequent addition of $0.02 \mathrm{~mL}$ of linoleic acid and $0.2 \mathrm{~mL}$ of Tween 40 . Plant extracts $(0.2 \mathrm{~mL})$ were added in the prepared mixture and a control was also run. The resultant mixtures were incubated at $20^{\circ} \mathrm{C}$ for 15 mins. Chloroform was removed using the rotary evaporator at $40^{\circ} \mathrm{C}$ and $50 \mathrm{~mL}$ of distilled water was added. The obtained mixture was shaken for 2 mins to form emulsion. The absorbance for all samples was measured immediately ( 0 time) and after incubation of samples for $120 \mathrm{~min}$ at $50^{\circ} \mathrm{C}$.

$$
\mathrm{AA} \%=[1-(\mathrm{Ao}-\mathrm{At}) /(\mathrm{Co}-\mathrm{Ct})] \times 100
$$

Where Ao and Co are the absorbance of sample and control respectively at 0 time. The At and $\mathrm{Ct}$ are absorbance values of sample and blank after 120 mins of incubation. Positive control contained BHA under same set of conditions.

\subsection{Fe-chelating activity}

Fe-chelating activity of hyroethanolic leaf extracts was determined following the reported method by Dinis et al. (1994). Briefly, $5 \mathrm{mM}$ solution of ferrozine reagent was prepared and $0.2 \mathrm{~mL}$ from this were mixed with 100 $\mu \mathrm{L}$ of $2 \mathrm{mM} \mathrm{FeSO}_{4}$. The methanolic plant extracts at concentration of $2 \mathrm{mg} / \mathrm{mL}$ were mixed with reaction mixture followed by an incubation of 10 mins. The absorbance was measured at $562 \mathrm{~nm}$ for each sample. Following equation was used to calculate the chelating activity

Fe-chelating activity $\%=\left(\mathrm{A}_{\text {control }}-\mathrm{A}_{\text {sample }}\right) / \mathrm{A}_{\text {control }} \times 100$

The control sample contained the all reagents except plant extract, where EDTA was used as positive control.

\subsection{Pancreatic lipase inhibition}

In-vitro anti-obesity activity of $C$. erectus leaf extracts was performed by measuring the inhibition of pancreatic lipase enzyme. Plant extracts were added to $0.01 \mathrm{M}$ Tris- $\mathrm{HCl}$ buffer along with pancreatic lipase. Olive oil was mixed with gum Arabic (dissolved in 0.1 $\mathrm{M}$ Tris- $\mathrm{HCl}$ buffer having $\mathrm{pH} 8.0,20 \mathrm{mM} \mathrm{CaCl}_{2}$ and 0.5 $\mathrm{M} \mathrm{NaCl}$ ). The lipase inhibition of plant extracts was determined following the previously reported method by Fukumoto et al. (1963) with slight modification. To carry out the procedure, $0.5 \mathrm{~mL}$ of each plant extract was mixed with $0.2 \mathrm{~mL}$ of lipase solution and allowed to stand for 30 minutes at $4^{\circ} \mathrm{C}$ to allow the reaction to occur completely; following which $2 \mathrm{~mL}$ of the substrate was added. The reaction mixture was incubated for 30 mins at $37^{\circ} \mathrm{C}$. The reaction was stopped by adding ethanol and acetone in 1:1 composition and the released free fatty acids (FFA) were titrated against $0.02 \mathrm{M} \mathrm{NaOH}$ till the $\mathrm{pH}$ became 9.4. All the measurements were performed in triplicates and percent inhibition was calculated by the following equation:

$$
\% \text { Inhibition }=100 \%-[(\mathrm{Vs} / \mathrm{Vc}) \times 100]
$$

Where, $100 \%$ is the enzymatic activity of control. The Vs and Vc represent the volume of base used in titration for sample and control, respectively.

\section{$2.6{ }^{1} H$-NMR based prediction of metabolites}

The most active fraction was subjected to metabolite 
prediction. Initially, $25 \mathrm{mg}$ of $C$. erectus most potent leaf extract were mixed with $\mathrm{CH}_{3} \mathrm{OH}-d_{4}$ (no internal standard) and with $0.375 \mathrm{~mL}$ of $\mathrm{KH}_{2} \mathrm{PO}_{4}$ buffer $(\mathrm{pH}=60)$ in $\mathrm{D}_{2} \mathrm{O}$ containing TSP $(0.1 \%)$. After 1 minute of vortex, ultrasonication was made for 15 mins at $35^{\circ} \mathrm{C}$. The solution was subjected to centrifugation at $13000 \mathrm{rpm}$ for 10 mins. Supernatant in amount of $600 \mu \mathrm{L}$ was collected in NMR tube and ${ }^{1} \mathrm{H}-\mathrm{NMR}$ analysis was performed. INOVA $500 \mathrm{MHz}$ spectrometer (Varian Inc, CA), at $499.887 \mathrm{MHz}$ frequency was used for spectrum generation at $26^{\circ} \mathrm{C}$ with tetramethylsilane (TMS) was the internal standard. The spectrum was bucketed with MestRenova 11.0 and Chenomyx software.

\subsection{Statistical analysis}

The findings were subjected to analysis of variance with Tukey's test to evaluate the level of significant difference. Minitab 17.0 statistical software was used. The analyses were carried out in triplicate.

\section{Results}

\subsection{Total antioxidant power}

The antioxidant potential of plant extracts is represented in Figure 1. High values of absorbance indicated good antioxidant potential of extracts. The $60 \%$ ethanolic extract was the most promising one in terms of latent antioxidant capacity having TAP of $223.0 \pm 3.27$ mg ASE/g PE but less than exhibited by BHA (309.16 $\pm 3.90 \mathrm{mg}$ ASE $/ \mathrm{g} \mathrm{PE}$ ). The aqueous extract exhibited lowest TAP value i.e., $47.33 \pm 2.88 \mathrm{mg}$ ASE/g $\mathrm{PE}$ among all fractions.

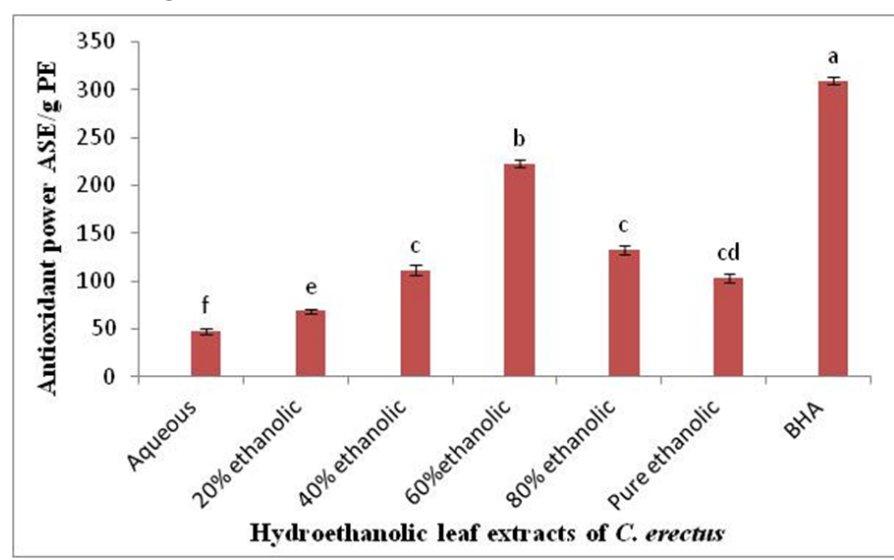

Figure 1. Total antioxidant power of $C$. erectus leaf extracts in comparison with BHA. Different alphabets on the bars are statistically significant at $\rho<0.05$.

Statistical analysis indicated significant level of difference $(\rho<0.05)$ among the TAP values of $60 \%$ ethanolic fraction when compared with remaining fractions. The pure ethanolic, $80 \%$ and $40 \%$ ethanolic fractions were statistically non-significant.

\section{$3.2 \beta$-carotene bleaching assay}

The comparative evaluation of hydroethanolic leaf extracts of $C$. erectus ranked $60 \%$ extract as the most efficient to hinder the bleaching of $\beta$-carotene (Figure 2).

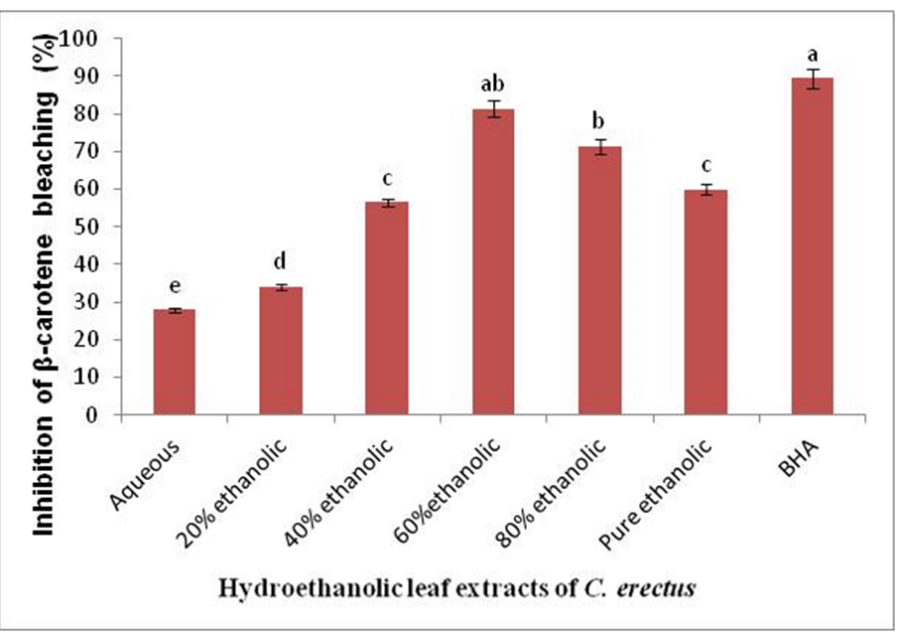

Figure 2. Comparative inhibition of $\beta$-carotene bleaching by C. erectus leaf extracts. Different alphabets on the bars are statistically significant at $\rho<0.05$.

The percentage peroxide inhibition for $60 \%$ ethanolic extract was $81.39 \pm 2.11 \%$ being statistically non-significant from BHA $(89.41 \pm 2.45 \%)$ which is a strong and efficient synthetic antioxidant $(\rho<0.05)$. Further comparison elaborated that $60 \%$ ethanolic extract was associated with most promising antioxidant attributes among all other solvent fractions. This discriminatory status might be due to variable distribution of phytochemicals in extracts.

\subsection{Fe-chelating activity}

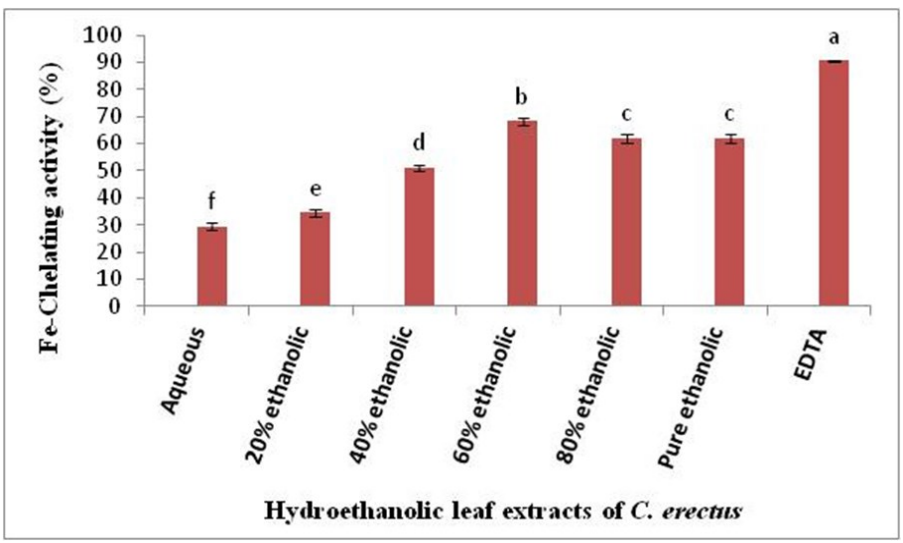

Figure 3. Fe-chelating activity of $C$. erectus leaf extracts. Different alphabets on the bars are statistically significant at $\rho<0.05$.

The results of Fe-chelating activity are presented in Figure 3. The $60 \%$ ethanolic extract of C. erectus showed maximum metal chelating activity of $68.21 \pm 1.17 \%$ in terms of inhibition percentage. The statistical analysis revealed that the metal chelating activity for $60 \%$ ethanolic extract was significantly higher than the other respective extracts $(\rho<0.05)$.

\subsection{Pancreatic lipase inhibition}


The current investigation regarding PLI presented $60 \%$ ethanolic extract as the most efficient anti-obesity fraction followed by $80 \%$ ethanolic extract. Statistical analysis indicated the significant level of difference among the percentage inhibition values of extracts, however the PLI for $40 \%$ and $80 \%$ ethanolic extracts was statistically non-significant $(\rho<0.05)$. The standard drug orlistat showed maximum PLI with value of $66.65 \pm 0.68$ and no extract could match the standard drug (Figure 4).

However, PLI of $50.60 \pm 1.47 \%$ by $60 \%$ ethanolic extract was the highest among extracts and could be considered as promising and effective.

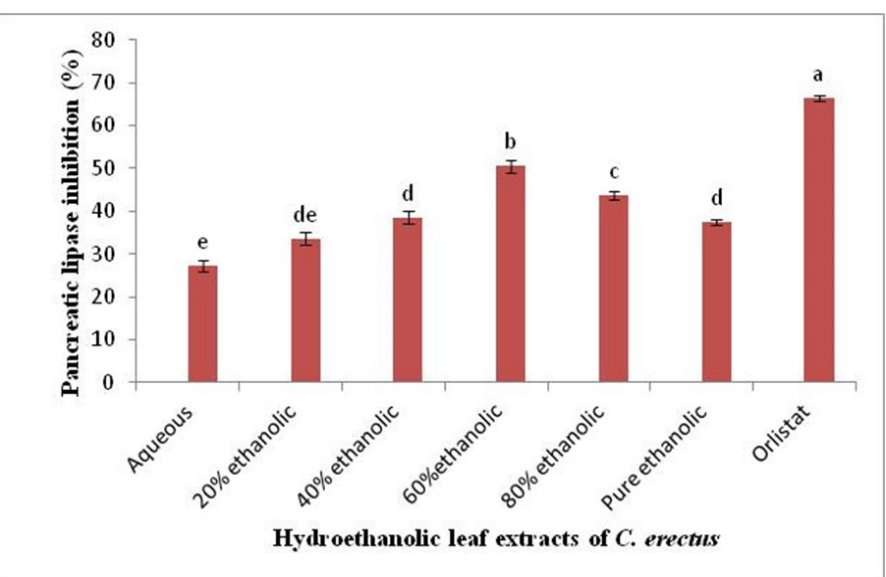

Figure 4. Pancreatic lipase inhibitory action of C. erectus leaf extracts and orlistat. Different alphabets on the bars are statistically significant at $\rho<0.05$.

\section{$3.5^{1} \mathrm{H}$-NMR based prediction of metabolites}

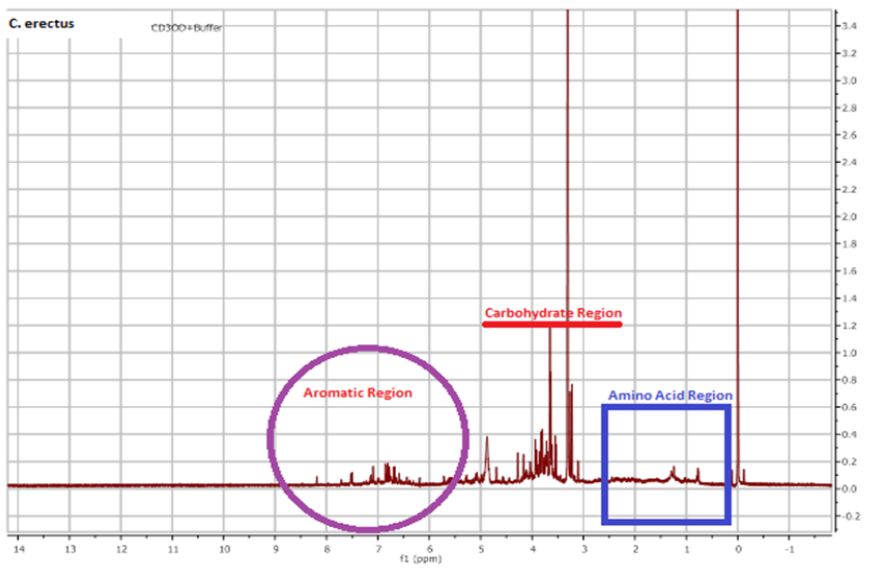

Figure 5. The ${ }^{1} \mathrm{H}-\mathrm{NMR}$ spectrum of $60 \%$ ethanolic leaf extract of $C$. erectus.

The ${ }^{1} \mathrm{H}$-NMR spectrum of $60 \%$ ethanolic extracts of $C$. erectus is represented as Figure 5 . The chemical shift values $\left(\delta_{\mathrm{H}}\right)$ corresponded to the nature of metabolites present in leaf extract were highlighted in the spectrum. The ${ }^{1} \mathrm{H}-\mathrm{NMR}$ spectrum is split into aromatic region and aliphatic region. The aromatic region corresponds to the peaks associated with protons of phenolic regime while aliphatic region is used for primary metabolites like sugars, amino acids and fatty acids. The regions ranging from $\delta_{\mathrm{H}} 6-9 \mathrm{ppm}$ is defined as aromatic region and is used for the identification of polyphenols including flavonoids. The region having $\delta_{\mathrm{H}} 3.5 \mathrm{ppm}$ to $4 \mathrm{ppm}$ and 1-2.5 ppm were categorized as carbohydrate and organic acid regions, respectively. Organic acid region may have peaks for amino acids and fatty acids; however, this region is typically named as amino acid region (Mediani et al., 2012; Mediani et al., 2015).

\section{Discussion}

Antioxidant activity is a basic and preliminary tool to assess the possible medicinal potential of plants and metabolites. The $60 \%$ ethanolic leaf extract of $C$. erectus exhibited highest in-vitro antioxidant activities. The $\beta$ carotene bleaching based antioxidant potential of $60 \%$ ethanolic extract was much higher than recently reported inhibition percentage of $17.88 \pm 3.13$ for ethanolic extract of Bromelia laciniosa (de Oliveira-Júnior et al., 2017). The elevated concentrations of extracts exhibited high antioxidant potential which might be due to higher contents of bioactive constituents mainly phenolic and flavonoids, generally responsible for antioxidant, antiradical and antidiabetic properties of plants (Raza et al., 2013; Evary et al., 2018). Plants are rich sources of natural lipase inhibitors, and hence may provide a workable choice to control obesity along with other associated disorders like diabetes (Jaradat et al., 2017). The lipid metabolism plays a prominent role in energy homeostasis of body. Dietary intake of high fat and highcalorie diets are responsible for weight gain and fat deposition. Lipase enzyme hydrolyzes triglycerides in intestine and facilitates their absorption in body leading to high-fat incorporation. It is very difficult to amend the existing lifestyle and prevailing dietary habits to a great extent for obesity management. On the other hand, complimentary use of synthetic anti-obesity drugs is a matter of concern due to their questionable safety. Under such circumstances, the natural lipase inhibitors are valuable tool for obesity management and plants can serve the purpose smoothly and effectively. The inhibition of pancreatic lipase by plant extracts was probably due to the structural interaction among bioactive metabolites and enzymes. The biologically active substances in extracts may bind with the active sites of pancreatic lipase to modify its functions. Such functional modifications result in reduced enzymatic activity which in turn controls the post-prandial lipidemia (Martinez-Gonzalez et al., 2017).

Iron is required by human body for routine body function but sometimes its excess may be harmful to living system. The ROS production due to iron load may lead to diabetes mellitus making iron load as an important reason behind disease pathogenesis. The exact 
mode of action of iron in DMT2 initiation is not fully known but it has been reported that excess iron may damage beta cell of pancreas and also develop insulin insensitivity (Simcox and McClain, 2013). Similarly, the function of liver was also reported to be disturbed under iron load conditions which in turn affected glucose homeostasis and iron extraction (Britton et al., 2018). The Fe-chelating activity by plants extracts indicated that this capacity may be another mechanized reason behind the antidiabetic role of $C$. erectus leaves.

The qualitative prediction of metabolites in $60 \%$ ethanolic extract (most potent) was performed by ${ }^{1} \mathrm{H}-$ NMR spectroscopy. The ${ }^{1} \mathrm{H}-\mathrm{NMR}$ based marking of primary and secondary metabolites has emerged as a novel and effective technique to move into metabolomics. Although, plant metabolomics is a very complicated field especially when plant extract is involved instead of a specific compound or fraction. Besides these tough lines, the ${ }^{1} \mathrm{H}-\mathrm{NMR}$ may be employed to confirm the presence of both primary and secondary plant metabolites (Mediani et al., 2012). The ${ }^{1} \mathrm{H}-\mathrm{NMR}$ technique is unbiased, non-destructive and needs no derivatization. This technique can be used for both qualitative and quantitative analysis of metabolites in crude plant extracts (Mediani et al., 2015). The primary metabolites mainly include carbohydrates and amino acids. Secondary metabolites are mainly, phenolic compounds, flavonoids, tannins and alkaloids. These metabolites produced characteristic peaks or signals due to protonic response to applied magnetic field in ${ }^{1} \mathrm{H}$ NMR spectrum and from these signals the nature of metabolites may easily be screened out. The numerous peaks in aromatic region confirmed the presence of secondary metabolites (polyphenols). The secondary metabolites are considered as functional tool to treat chronic ailments including diabetes and obesity.

The antioxidant activity, Fe-chelating activity and pancreatic lipase inhibition potential suggested the $C$. erectus leaves as a novel source of value able natural metabolites which were confirmed by ${ }^{1} \mathrm{H}-\mathrm{NMR}$ response. The $C$. erectus may be exploited to expedite the development in field of nutraceuticals and functional foods.

\section{Conclusion}

The $60 \%$ ethanolic leaf extract of $C$. erectus was proved as the most effective fraction to inhibit bleaching of $\beta$-carotene, chelation of iron and inhibition of pancreatic lipase. The ${ }^{1} \mathrm{H}-\mathrm{NMR}$ predicted the presence of various important classes of metabolites including polyphenols and organic acids in leaf extract. The outcomes of this work may be extended further for in vivo trials and toxicity evaluation to move into regime of nutraceuticals and functional foods with antioxidant and antiobesity attributes.

\section{Conflict of Interest}

It is declared that authors have no conflict of interest.

\section{References}

Britton, L., Bridle, K., Reiling, J., Santrampurwala, N., Wockner, L., Ching, H., Stuart, K., Subramaniam, V.N., Jeffrey, G., St. Pierre, T., House, M., Gummer, J., Trengove, R., Olynyk, J., Crawford, D. and Adams, L. (2018). Hepatic iron concentration correlates with insulin sensitivity in nonalcoholic fatty liver disease. Hepatology Communications, 2 (6), 644-653. https://doi.org/10.1002/hep4.1190

de Oliveira-Júnior, R.G., Ferraz, C.A.A., Souza, G.R., Guimarães, G.R.S.A., de Oliveira, A.P., de LimaSaraiva, S.R.G., Rolim, L.A., Rolim-Neto, P.J. and da Silva Almeida, J.R.G. (2017). Phytochemical analysis and evaluation of antioxidant and photoprotective activities of extracts from flowers of Bromelia laciniosa (Bromeliaceae). Biotechnology and Biotechnological Equipment, 31(3), 600-605. https://doi.org/10.1080/13102818.2017.1288073

Dinis, T.C., Madeira, V.M. and Almeida, L.M. (1994). Action of phenolic derivatives (acetaminophen, salicylate, and 5-aminosalicylate) as inhibitors of membrane lipid peroxidation and as peroxyl radical scavengers. Archives of Biochemistry and Biophysics, 315(1), 161-169. https://doi.org/10.1006/ abbi.1994.1485

Evary, Y.M.,Nugroho, A.E. and Pramono, S. (2018). Comparative study on DPPH free radical scavenging and alpha-glucosidase inhibitory activities of ethanolic extracts from different parts of durian plant (Durio zibethinus Murr.). Food Research, 3(5), 463 - 468. https://doi.org/10.26656/fr.2017.3(5).085

Fukumoto, J., Tsujisaka, Y. and Iwai, M. (1963). STUDIES ON LIPASE. The Journal of General and Applied Microbiology, 9(3), 353-361. https:// doi.org/10.26656/fr.2017.3(5).085

Jaradat, N., Zaid, A., Hussein, F., Zaqzouq, M., Aljammal, H. and Ayesh, O. (2017). Anti-lipase potential of the organic and aqueous extracts of ten traditional edible and medicinal plants in Palestine; a comparison study with orlistat. Medicines, 4(4), 89. https://doi.org/10.3390/medicines 4040089

Madhujith, T. and Shahidi, F. (2006). Optimization of the extraction of antioxidative constituents of six barley cultivars and their antioxidant properties. Journal of Agricultural and Food Chemistry, 54(21), 
8048-8057. https://doi.org/10.1021/jf061558e

Martinez-Gonzalez, A.I., Alvarez-Parrilla, E., DíazSánchez, Á.G., de la Rosa, L.A., Núñez-Gastélum, J.A., Vazquez-Flores, A.A. and Gonzalez-Aguilar, G.A. (2017). In vitro inhibition of pancreatic lipase by polyphenols: A kinetic, fluorescence spectroscopy and molecular docking study. Food Technology and Biotechnology, 55(4), 519-530. https://doi.org/10.17113/ftb.55.04.17.5138

Mediani, A., Abas, F., Khatib, A., Maulidiani, H., Shaari, K., Choi, Y.H. and Lajis, N. (2012). ${ }^{1} \mathrm{H}-$ NMR-based metabolomics approach to understanding the drying effects on the phytochemicals in Cosmos caudatus. Food Research International, 49(2), 763-770. https:// doi.org/10.1016/j.foodres.2012.09.022

Mediani, A., Abas, F., Khatib, A., Tan, C.P., Ismail, I.S., Shaari, K., Ismail, A. and Lajis, N.H. (2015). Phytochemical and biological features of Phyllanthus niruri and Phyllanthus urinaria harvested at different growth stages revealed by $1 \mathrm{H}$ NMR-based metabolomics. Industrial Crops and Products, 77, 602-613. https://doi.org/10.3390/ molecules 22060902

Nascimento, D.K., Souza, I.A., OLiveira, A.F.D., Barbosa, M.O., Santana, M.A., Pereira Junior, D.F., Lira, E.C. and Vieira, J.R. (2016). Phytochemical screening and acute toxicity of aqueous extract of leaves of Conocarpus erectus Linnaeus in swiss albino mice. Anais da Academia Brasileira de Ciências, 88(3), 1431-1437. https:// doi.org/10.1590/0001-3765201620150391

Piyush, G., Mendhe, B., Namrata, S., Milind, P., Anupam, P. and Sahadev, Y. (2010). Antioxidant activity of flavonoidal content of Butea monosperma. International Journal of Pharmaceutical Sciences and Research (IJPSR), 1 (4), 85-88. http://dx.doi.org/10.13040/IJPSR.09758232.1(4).85-88

Prieto, P., Pineda, M. and Aguilar, M. (1999). Spectrophotometric quantitation of antioxidant capacity through the formation of a phosphomolybdenum complex: specific application to the determination of vitamin E. Analytical Biochemistry, 269(2), 337-341. https:// doi.org/10.1006/abio.1999.4019

Raza, S.A., Chaudhary, A.R., Mumtaz, M.W., Ghaffar, A., Adnan, A. and Waheed, A. (2018). Antihyperglycemic effect of Conocarpus erectus leaf extract in alloxan-induced diabetic mice. Pakistan Journal of Pharmaceutical Sciences, 31(2 Suppl), 637-642.

Raza, S.A., Rashid, A., William, J., Arshed, S.F. and
Arshad, M. (2013). Comparison of antioxidant activity of some medicinally important plants from Pakistan. Acta Scientiarum Polonorum Technologia Alimentaria, 12(4), 403-410.

Saleh, E.-S., Bazaid, S., Shohayeb, M., El-Sayed, M. and El-Wakil, E. (2012). Phytochemical studies and evaluation of antioxidant, anticancer and antimicrobial properties of Conocarpus erectus L. growing in Taif, Saudi Arabia. European Journal of Medicinal Plants, 2(2), 93-112. https:// doi.org/10.1155/2013/345465

Saleh, E.-S., Bazaid, S.A. and Sbara, A.-N. (2013). Protective effect of conocarpus erectus extracts on CCl4-induced chronic liver injury in mice. Global Journal of Pharmacology, 7, 52-60.

Shaaban, M.A., Dawod, A.E.A. and Nasr, M.A. (2016). Role of iron in diabetes mellitus and its complications. Menoufia Medical Journal, 29(1), 1116. https://doi.org/10.4103/1110-2098.178938

Shon, M.Y., Kim, T.H. and Sung, N.J. (2003). Antioxidants and free radical scavenging activity of Phellinus baumii (Phellinus of Hymenochaetaceae) extracts. Food Chemistry, 82(4), 593-597. https:// doi.org/10.1016/S0308-8146(03)00015-3

Simcox, J.A. and McClain, D.A. (2013). Iron and diabetes risk. Cell Metabolism, 17(3), 329-341. https://doi.org/10.1016/j.cmet.2013.02.007

Wang, J., Zhang, Q., Zhang, Z., Song, H. and Li, P. (2010). Potential antioxidant and anticoagulant capacity of low molecular weight fucoidan fractions extracted from Laminaria japonica. International Journal of Biological Macromolecules, 46(1), 6-12. https://doi.org/10.1016/j.ijbiomac.2009.10.015

William, J., John, P., Mumtaz, M. W., Ch, A. R., Adnan, A., Mukhtar, H., Sharif, S. and Raza, S.A. (2018). Antioxidant activity, Hypoglycemic potential and metabolite profiling of Hyophorbe indica leaf extract. Pakistan Journal of Pharmaceutical Sciences, 31(6 Suppl.), 2737-2742. 